\title{
Natural history of angiomyolipoma in lymphangioleiomyomatosis: implications for screening and surveillance
}

Zhao W Yeoh ${ }^{1}$, Vidya Navaratnam², Rupesh Bhatt ${ }^{3}$, lan McCafferty ${ }^{3}$, Richard B Hubbard ${ }^{2}$ and Simon R Johnson ${ }^{1,4^{*}}$

\begin{abstract}
Background: LAM is a rare disease of women categorised by lung cysts and lymphatic abnormalities. The disease occurs sporadically or associated with Tuberous Sclerosis Complex (TSC-LAM). Angiomyolipoma, a benign tumour, prone to haemorrhage, occurs mostly in the kidneys in many of these patients. Treatment guidelines exist for angiomyolipoma in patients with TSC but the natural history of angiomyolipoma in sporadic LAM has not been studied.
\end{abstract}

Aims: To document the natural history of angiomyolipoma in a national cohort of patients with sporadic LAM to inform tumour screening and surveillance protocols.

Methods: Demographic data, clinical features, lung function and tumour size were obtained from clinical records of patients attending the National Centre for LAM in Nottingham, UK.

Results: 122 patients with definite or probable LAM by European Respiratory Society criteria were identified. One hundred and seven had sporadic LAM, of which 53 (50\%) had at least one angiomyolipoma. In patients with sporadic LAM presentation of angiomyolipoma preceded or followed onset of lung symptoms by up to 11 and 38 years respectively. Mean tumour size was $28 \mathrm{~mm}$ (range 5-140 mm) at presentation and growth was $1.8 \mathrm{~mm} / \mathrm{yr}$ (95\% C.I. 0.42-3.82) thereafter. Eleven patients with sporadic LAM had had a nephrectomy due to angiomyolipoma bleeding. The need for intervention did not differ between those with TSC-LAM and sporadic LAM.

Conclusions: Patients with LAM have a high prevalence of symptomatic angiomyolipoma which can present at any time. Angiomyolipoma in sporadic-LAM have a similar risk of bleeding to those with TSC. All patients should be screened for angiomyolipoma at diagnosis of lung disease by MRI scanning and the tumours require continuous monitoring.

Keywords: Lymphangioleiomyomatosis, Tuberous sclerosis, Kidney disease, Natural history

\section{Introduction}

Lymphangioleiomyomatosis (LAM) is a rare systemic disease, almost exclusively affecting women with a prevalence of $5-10 /$ million women [1]. LAM causes lung cysts and lymphatic abnormalities leading to recurrent pneumothorax, respiratory impairment and chylous collections [2]. LAM occurs both sporadically and in patients with tuberous sclerosis complex (TSC). Angiomyolipoma, a

\footnotetext{
* Correspondence: simon.johnson@nottingham.ac.uk

'Divisions of Respiratory Medicine and Respiratory Research Unit, School of Medicine, University of Nottingham, D Floor, South Block. Queens Medical Centre, Nottingham NG7 2UH, UK

${ }^{4}$ National Centre for Lymphangioleiomyomatosis, Nottingham University Hospitals NHS Trust, Nottingham, UK

Full list of author information is available at the end of the article
}

benign tumour, is present in up to half of patients with sporadic LAM $[3,4]$ and nearly all patients with TSCLAM $[5,6]$.

The majority of angiomyolipomas do not cause symptoms although larger tumours are at risk of bleeding. Failure to identify and prophylactically treat larger tumours with either selective embolisation or nephron sparing surgery can result in retroperitoneal haemorrhage and sometimes a nephrectomy [7]. More recently, pharmacologic inhibition of the kinase mTOR has been shown to reduce angiomyolipoma volume in patients with TSC and sporadic LAM [8-10] and mTOR inhibitors are now recommended for treatment of angiomyolipoma in TSC [11]. 
Guidelines for management of angiomyolipoma are based on case series $[12,13]$ and suggest that angiomyolipoma in patients with TSC grow more rapidly and are more prone to complications than angiomyolipoma in non-TSC patients [7]. However data on the natural history of angiomyolipoma in sporadic LAM are very sparse and it is not clear if angiomyolipoma in these patients really are less prone to haemorrhage and should be treated differently from those with TSC. We have examined the clinical characteristics, growth rate and complications of angiomyolipoma in women with sporadicLAM to optimise screening protocols for these patients.

\section{Methods}

\section{Patients}

Patients were recruited from the National Centre for LAM in Nottingham UK, a referral centre providing comprehensive care for both sporadic and TSC-LAM. All patients were over the age of 18 years and had either definite or probable LAM according to European Respiratory Society (ERS) criteria [14]; those with possible LAM were excluded. TSC was diagnosed according to current criteria [15]. All patients had a clinical examination looking for signs of TSC including a dermatologic examination with a Woods light. In keeping with the ERS LAM guidelines; where TSC could not be excluded clinically, patients were evaluated by a clinical geneticist. Ethical approval was obtained from the Trent MultiCentre Research Ethics Committee (NRES 07/H0403/ 165 and NRES 05/Q2403/187) and all patients provided informed consent. Patient histories were taken in a standardised format for clinical purposes and baseline assessment included screening for TSC as recommended in the ERS LAM guidelines [14]. Lung function tests were measured according to ERS/British Thoracic Society standards as part of clinical care [16]. Lymphatic involvement was evaluated at baseline and defined as the presence of chylous collections in the abdomen or thorax, abdominal or pelvic lymphadenopathy, diffuse lymphatic enlargement or lymyphangioleiomyomas visible by CT scanning. Renal history and imaging was obtained from both the referring centre and investigations performed at the National LAM Centre. Angiomyolipoma imaging was performed for routine clinical care according to the LAM Centre angiomyolipoma protocol where patients have cross sectional imaging of their renal tract, at or as soon after diagnosis as practical, and for those with angiomyolipoma, a measurement of tumour size at yearly intervals thereafter. Tumour size measurements obtained prior to attending the LAM Centre were performed at the referring clinician's discretion. Tumour size was expressed as the longest diameter of angiomyolipomas measured by either ultra-sound (US), computerised tomography $(\mathrm{CT})$ or magnetic resonance imaging (MRI) as previously described (Figure 1) [9]. Measurements were performed by either the reporting radiologist, or a member of the study team (SJ). Unquantifiable small tumours were assigned a measurement of $5 \mathrm{~mm}$. Tumour measurements post angiomyolipoma surgery, post embolization or when using mTOR inhibitors were excluded from the analysis of tumour growth.

\section{Analysis}

Groups were compared using linear regression, chi square or t-tests. Tumour growth rate $(\mathrm{mm} / \mathrm{yr})$ was defined as the slope of a regression line fitted to tumour measurements for patients who had repeated imaging. Tumour size categories were compared by a 1 way analysis of variance (ANOVA). Time to intervention was analysed by Kaplan-Meier analysis. Intervention was defined as a composite endpoint comprising action taken in response to spontaneous tumour bleeding, use of an mTOR inhibitor for angiomyolipoma, or referral to an interventional service at the discretion of the supervising clinician. Calculations were performed using Graphpad Prism version 6.00, (GraphPad Software, San Diego California USA) and Stata v11 (Texas)).

\section{Results}

\section{Patient cohort}

One hundred and twenty two patients with definite or probable LAM by ERS criteria were identified [14]. One hundred and seven patients had sporadic LAM and 15 TSC-LAM. Of those with sporadic LAM, the mean age at diagnosis with respiratory disease was 35.3 years (SD 10.4). The mean age at the time of the study was 50 years

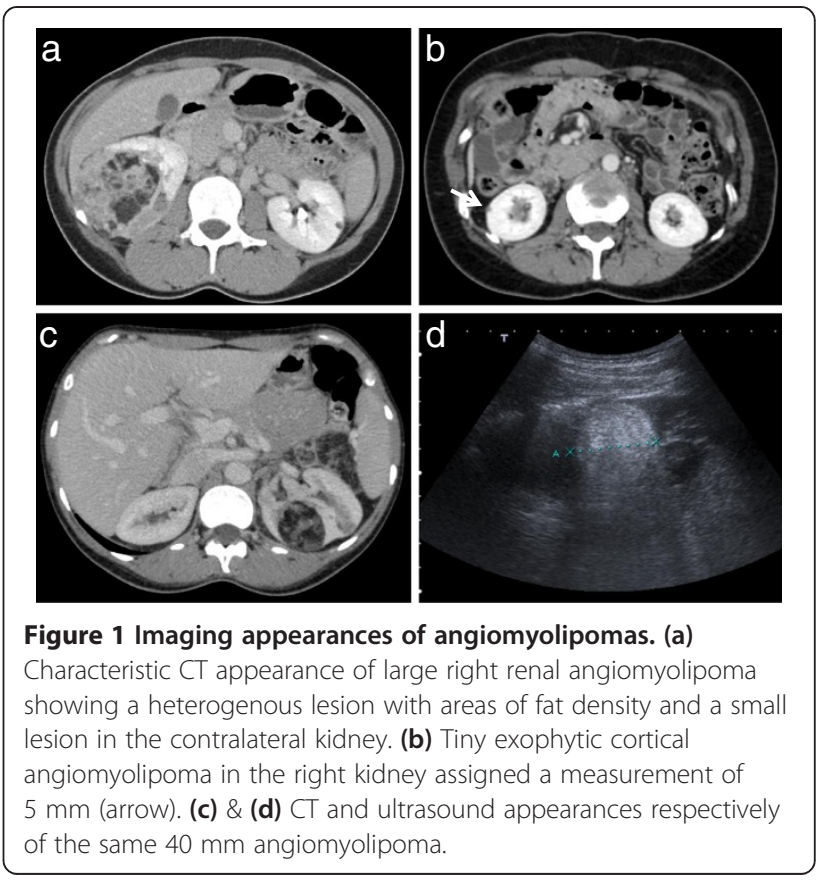


(SD 11.6). Presentation of respiratory disease was due to shortness of breath in 53\%, pneumothorax in $28 \%$, cough in $4 \%$, and haemoptysis in $3 \%$. The remaining $12 \%$ presented with either extra-pulmonary manifestations or were found to have LAM after investigation for unrelated problems.

\section{Presentation with angiomyolipoma}

Of the 107 patients with sporadic LAM, 53 (50\%) had, or had had, at least one renal angiomyolipoma. Two patients with renal angiomyolipoma had co-existent hepatic angiomyolipomas. Hepatic angiomyolipomas were not included in the analysis. The mean age at discovery of angiomyolipoma was 39.1 years (SD 13.2). Nineteen out of $53(36 \%)$ of those with angiomyolipoma presented due to symptoms. In 11 patients this was abdominal or flank pain, in three haematuria, in three palpable mass and in two abdominal bloating. Of these patients presenting with angiomyolipoma symptoms, 11 were treated by nephrectomy, including one with renal bleeding during pregnancy. The median age at nephrectomy was 23 (range 13-49). Nine of these 11 had bilateral angiomyolipoma. The remaining $64 \%$ of angiomyolipomas were detected by screening after the diagnosis of lung disease (Table 1).

In 10 patients detection of angiomyolipoma preceded the diagnosis of lung disease by up to 11 years. In 10, the diagnosis of lung disease was made at the time of renal presentation but in the remainder, angiomyolipoma was detected up to 38 years after the onset of respiratory symptoms (Figure 2). Reliable tumour measurements for patients treated with nephrectomy at diagnosis were not generally available and could not be included in tumour size

Table 1 Characteristics of patients with or without angiomyolipoma

\begin{tabular}{|c|c|c|c|c|}
\hline & \multicolumn{3}{|c|}{ Sporadic-LAM } & \multirow[t]{3}{*}{ TSC-LAN } \\
\hline & \multicolumn{2}{|c|}{ Angiomyolipoma } & \multirow[b]{2}{*}{$p$ value } & \\
\hline & Present & Absent & & \\
\hline Number of patients & 53 & 54 & & 15 \\
\hline $\begin{array}{l}\text { Mean age at respiratory } \\
\text { presentation }{ }^{*} \text { (years) }\end{array}$ & 32.3 & 37 & 0.18 & 31.8 \\
\hline $\begin{array}{l}\text { Mean disease duration } \\
\text { (years) }\end{array}$ & 14.4 & 15.2 & 0.21 & 10.5 \\
\hline Ever had pneumothorax ${ }^{\#}(\%)$ & $16(32)$ & $13(24)$ & 0.37 & 40 \\
\hline $\begin{array}{l}\text { Lymphatic disease } \\
\text { detected }^{\#}(\%)\end{array}$ & $6(11)$ & 10 (19) & 0.036 & 13 \\
\hline Ever been pregnant ${ }^{\#}(\%)$ & $29(55)$ & $24(44)$ & 0.07 & 33 \\
\hline $\begin{array}{l}\text { Mean baseline } \mathrm{FEV}_{1}{ }^{*} \\
\text { (\% predicted) }\end{array}$ & 65.7 & 71.4 & 0.37 & 64 \\
\hline $\begin{array}{l}\text { Mean baseline } \mathrm{TL}_{\mathrm{CO}}{ }^{*} \\
\text { (\% predicted) }\end{array}$ & 57.7 & 59.3 & 0.79 & 47 \\
\hline
\end{tabular}

$\mathrm{p}=$ angiomyolipoma present vs. absent for patients with sporadic LAM.

Angiomyolipoma was present in all patients with TSC-LAM.

p value from $t$ test.

\#p value from chi squared test.

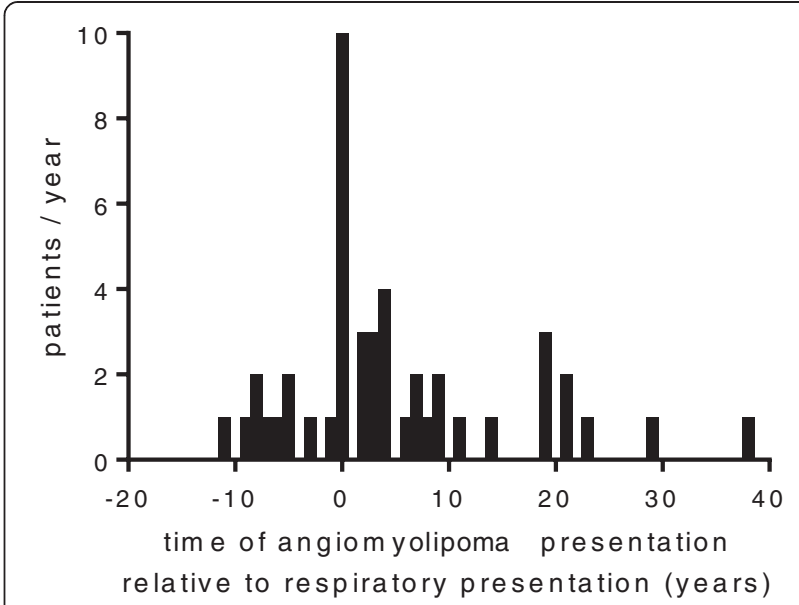

Figure 2 Angiomyolipoma may present at any point in the disease course. Frequency distribution of angiomyolipoma presentation relative to presentation with respiratory disease (respiratory diagnosis is at 0 years). Detection of angiomyolipoma may have been due to symptomatic disease or screening.

data. At presentation, the mean tumour size for patients with sporadic LAM was $28 \mathrm{~mm}$ (SD 12.6, Table 2). The presence of angiomyolipoma was not associated with duration of disease $(\mathrm{p}=0.72)$ or the degree of loss of $\mathrm{FEV}_{1}(\mathrm{p}=0.37)$. Patients with angiomyolipoma were less likely to have lymphatic involvement than those without angiomyolipoma $(\mathrm{p}=0.036)$ but other clinical features did not differ significantly between groups (Table 1).

Angiomyolipoma were present in all patients with TSC-LAM and significantly more common than in those with sporadic-LAM $(\mathrm{p}<0.0001$, Table 2$)$. Compared with TSC-LAM, patients with sporadic LAM tended to have smaller tumours which were less often bilateral. However there was no significant difference in the incidence

Table 2 Comparison of angiomyolipoma characteristics in patients with sporadic and TSC-LAM

\begin{tabular}{|c|c|c|c|}
\hline & Sporadic-LAM & TSC-LAM & $p$ value \\
\hline Total number of patients & 107 & 15 & \\
\hline $\begin{array}{l}\text { Patients with } \\
\text { angiomyolipoma }{ }^{\#}(\%)\end{array}$ & 50 & 100 & $<0.0001$ \\
\hline $\begin{array}{l}\text { Mean age at renal } \\
\text { presentation }^{*} \text { (years) }\end{array}$ & 39 & 38 & NS \\
\hline $\begin{array}{l}\text { Mean tumour size at } \\
\text { presentation }^{*}(\mathrm{~mm})\end{array}$ & 29 & 61 & 0.006 \\
\hline Bilateral tumours ${ }^{\#}\left(\%^{\dagger}\right)$ & 40 & 84 & 0.004 \\
\hline $\begin{array}{l}\text { Any renal tumour } \\
\text { symptoms }{ }^{\#}\left(\%^{\dagger}\right)\end{array}$ & 49 & 40 & NS \\
\hline Nephrectomy ${ }^{\#}\left(\%^{\dagger}\right)$ & 21 & 13 & NS \\
\hline
\end{tabular}

$\mathrm{p}$ value from $t$ test. NS $=$ not significant.

\#p value from chi squared test.

tpercentage of those with angiomyolipoma. 
of bleeding or the need for intervention between the two groups (Table 2).

\section{Natural history of angiomyolipoma}

More than one tumour measurement was available for 31 tumours from 26 patients with sporadic LAM. The mean duration of follow up was 3.8 years (SD 2.25). Seventeen (55\%) tumours increased in size, nine (29\%) did not change and five (16\%) appeared to reduce in size (Figure 3a). The mean rate of growth for angiomyolipoma in sporadic LAM was $1.8 \mathrm{~mm} /$ year $(95 \%$ confidence interval 0.42-3.82). Although there was no overall relationship between tumour size and growth, there was a trend toward larger tumours growing more rapidly (Figure $3 \mathrm{~b}$ ) and only those $30 \mathrm{~mm}$ or larger grew by more than $10 \mathrm{~mm} / \mathrm{yr}$ (Figure 3c).
The median time from identification of angiomyolipoma to a clinically significant renal event, defined as referral for embolization or surgery, mTOR inhibitor treatment, bleeding or other tumour symptom, was 34 years for patients with sporadic LAM and 25 years for TSCLAM (range 0-43) (Figure 4). There was no significant difference in clinically significant renal events between those with sporadic or TSC-LAM.

\section{Discussion}

To our knowledge this is the first study to examine the natural history of angiomyolipoma in a large cohort of patients with sporadic LAM. Our aim was to understand the growth and incidence of bleeding in patients with sporadic LAM to develop better screening protocols for these tumours. Our findings show that angiomyolipomas

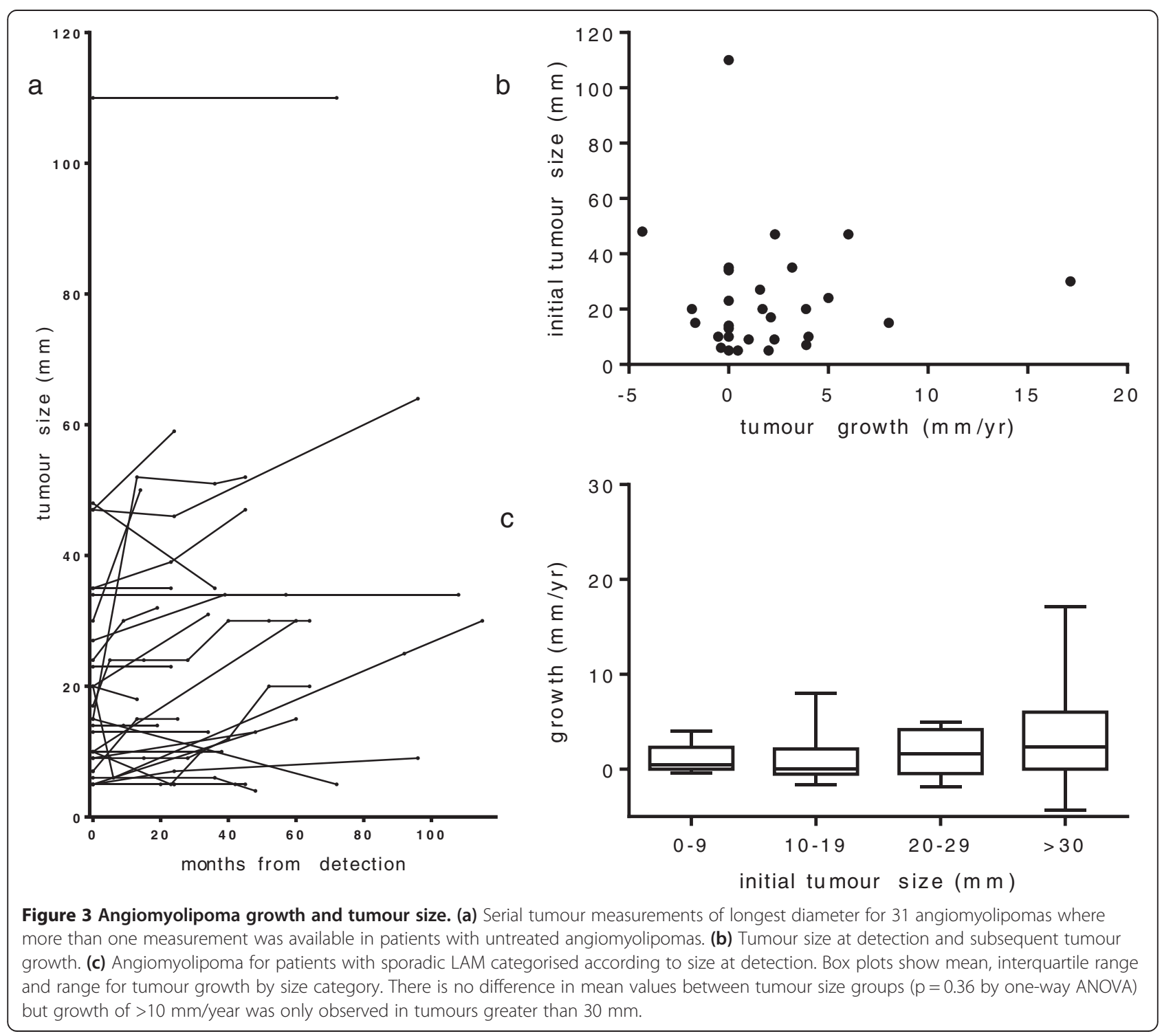




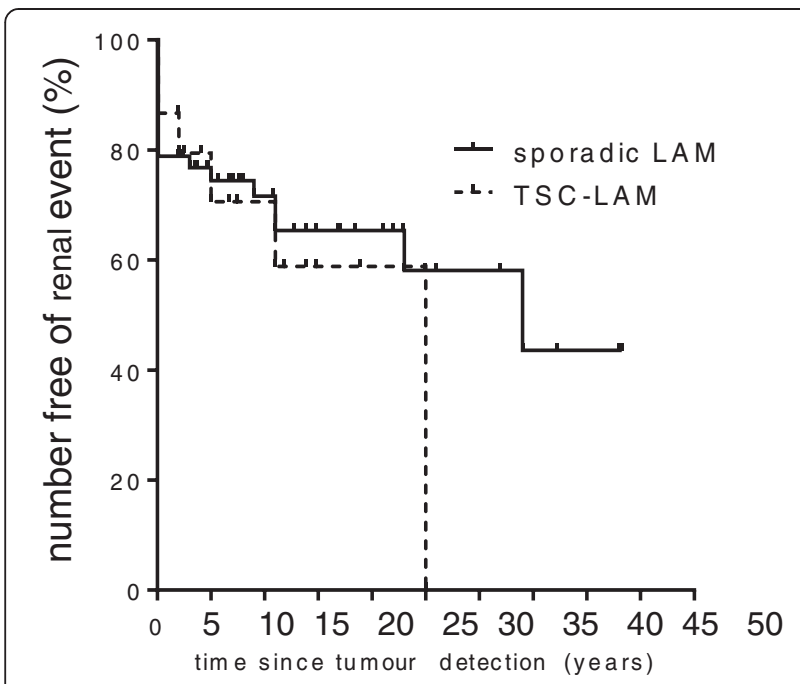

Figure 4 Time to need for intervention for angiomyolipoma.

Kaplan-Meier curves show the time to a significant renal event (categorised as spontaneous tumour bleeding, use of an mTOR inhibitor, or referral to an interventional service at the discretion of the supervising clinician) for patients with sporadic and TSC-LAM. There was no significant difference in the need for intervention between patients with sporadic and TSC-LAM.

are present in half of patients with sporadic LAM, can present at any time during the clinical course and are prone to growth and haemorrhage. Tumour presence cannot be predicted by the extent of the lung disease or other clinical features. This is important, as although patients with LAM are generally under regular follow up for lung disease, renal angiomyolipomas may be neglected and present with bleeding resulting in loss of renal function even in those with mild lung disease.

Previous series of angiomyolipoma may have underestimated the rates of TSC and LAM, particularly those with milder clinical features [17]. For this reason it has not been possible to address whether patients with nonTSC related angiomyolipoma are truly at lower risk of complications and should be treated differently from those with TSC. In contrast to previous studies, our findings suggest that patients with sporadic LAM and angiomyolipoma have a similar risk of complications to both historical series of those with TSC $[7,18,19]$ and with the small number of TSC-LAM patients within our cohort.

Eleven of 53 patients had a nephrectomy due to haemorrhage from an angiomyolipoma. Nephrectomy due to bleeding generally preceded the diagnosis of lung disease and the histological type of renal tumour was unknown at presentation. These patients had a mean age at nephrectomy of 23 years whereas the mean age at renal presentation of the cohort as a whole was 39 years and it is likely that these patients had more aggressive and rapidly growing tumours.
Despite this being one of the largest studies of the behaviour of angiomyolipoma, and the first to document growth over time, our findings need to be interpreted with some caution as some of these data are retrospective and some patients had had treatment for symptomatic tumours prior to enrolment. As these treated tumours were probably larger and more aggressive, overall growth rates and the incidence of complications may be underestimated. Furthermore, some outcome data are based upon surrogate endpoints such as referral for an intervention, which may have been influenced by factors other than tumour size alone. In the cohort studied, more patients with sporadic LAM were referred electively for consideration of an intervention than those with TSC. Additionally, data were collected using different imaging modalities and protocols which may impact upon the growth data, particularly where smaller lesions may be of similar size to the slice intervals used in cross sectional imaging. Further, as ultrasound is not as accurate for size measurements as CT or MRI and also less reliable at identifying the fat poor components of angiomyolipomas we attributed a value of $5 \mathrm{~mm}$ to all tumours with a longest diameter of $5 \mathrm{~mm}$ or less, and these issues may explain why some of the tumours appeared to shrink over time.

The study also examines only tumour size and does not account for the presence of vascular aneurysms which are also associated with bleeding [18]. Analysing the vascular anatomy of these tumours may be required to improve stratification of bleeding risk and can be done with both contrast CT and MRI.

\section{Implications for clinical practice}

Guidelines for patients with TSC have been recently published which recommend MRI of the abdomen to assess the progression of angiomyolipoma (and renal cystic disease) every 1-3 years throughout the lifetime of the patient. Intervention is recommended for tumours larger than three $\mathrm{cm}$ which is a more aggressive approach than that previously taken [11]. Patients with sporadic LAM are less prone to loss of renal function than those with TSC who commonly have multiple angiomyolipomas and may also have polycystic kidney disease. However, our findings suggest that patients with sporadic LAM are at significant risk of bleeding from angiomyolipoma, that these tumours may present at any point in the disease course, and grow unpredictably at a rate independent of progression of the lung disease. Although, from somewhat limited data, our findings suggest that only tumours greater than $30 \mathrm{~mm}$ are likely to grow in excess of $10 \mathrm{~mm} / \mathrm{yr}$ and reach a size of $40-45 \mathrm{~mm}$ before further imaging and thus be at greater risk of haemorrhage. We would therefore suggest that all patients are screened for the presence of angiomyolipoma at diagnosis by MRI. For those with tumours less than 
$10 \mathrm{~mm}$, imaging should be carried out every two years, for tumours between 11 and $30 \mathrm{~mm}$ in diameter, at 12 monthly intervals. For tumours greater than $30 \mathrm{~mm}$, repeat imaging should be performed at 6 monthly intervals or referred for consideration of an intervention at that point. All patients with angiomyolipoma should be warned of the symptoms of bleeding and imaging should be performed urgently in the presence of new symptoms possibly attributable to angiomyolipoma.

In summary, patients with sporadic LAM have a high prevalence of symptomatic angiomyolipoma which can present at any time during the course of the disease. Angiomyolipoma in sporadic LAM have a similar risk of bleeding to those with TSC and require continuous monitoring. Prospective evaluation of large cohorts of patients with LAM in the developing specialist services is required to improve understanding of the natural history, optimal screening protocols and risk stratification of these tumours.

\section{Notation of prior abstract publication/presentation}

Some data from the study were presented at the British Thoracic Society Winter Meeting, 2013.

\section{Abbreviations}

ANOVA: Analysis of variance; CT: Computerised tomography; ERS: European Respiratory Society; LAM: Lymphangioleiomyomatosis; MRI: Magnetic resonance imaging; mTOR: mammalian target of rapamycin; SD: Standard deviation; TSC: Tuberous Sclerosis Complex; US: Ultra-sound.

\section{Competing interest}

Prof Johnson has received grants from the MRC, BBSRC, British Lung Foundation, LAM Action and fees for speaking from Novartis. Prof Hubbard has received grants from the MRC and British Lung Foundation. Mr Bhatt has received fees for speaking from Novartis. The other authors report no conflict of interest.

\section{Authors' contribution}

Conception and design: SRJ, RBH. Data collection: ZWY, SRJ, VN. Analysis and interpretation: ZWY, SRJ, VN, RBH. Drafting the manuscript for important intellectual content: SRJ, ZWY, RB, IM, RBH. Guarantor for the study: SRJ. All authors read and approved the final manuscript.

\section{Funding}

ZWY was supported by a Wellcome vacation Scholarship. VN is funded by the NIHR as an Academic Clinical Fellow.

\section{Author details}

${ }^{1}$ Divisions of Respiratory Medicine and Respiratory Research Unit, School of Medicine, University of Nottingham, D Floor, South Block. Queens Medical Centre, Nottingham NG7 2UH, UK. Epidemiology and Public Health, School of Medicine, University of Nottingham, Nottingham, UK. ${ }^{3}$ School of Medicine, University of Nottingham, Renal tumour service, University Hospital Birmingham, Nottingham, UK. ${ }^{4}$ National Centre for

Lymphangioleiomyomatosis, Nottingham University Hospitals NHS Trust, Nottingham, UK.

Received: 14 July 2014 Accepted: 17 September 2014 Published online: 03 October 2014

\section{References}

1. Harknett EC, Chang WYC, Byrnes S, Johnson J, Lazor R, Cohen MM, Gray B, Geiling S, Telford H, Tattersfield AE, Hubbard RB, Johnson SR: Regional and national variability suggests underestimation of prevalence of lymphangioleiomyomatosis. Q J Med 2011, 104:971-979.
2. Johnson SR: Lymphangioleiomyomatosis. Eur Respir J 2006, 27:1056-1065.

3. Bernstein SM, Newell JD Jr, Adamczyk D, Mortenson RL, King TE Jr, Lynch DA: How common are renal angiomyolipomas in patients with pulmonary lymphangiomyomatosis? Am J Respir Crit Care Med 1995, 152:2138-2143.

4. Cohen MM, Pollock-BarZiv S, Johnson SR: Emerging clinical picture of lymphangioleiomyomatosis. Thorax 2005, 60:875-879.

5. Cudzilo CJ, Szczesniak RD, Brody AS, Rattan MS, Krueger DA, Bissler JJ, Franz DN, McCormack FX, Young LR: Lymphangioleiomyomatosis screening in women with tuberous sclerosis. Chest 2013, 144:578-585.

6. Rakowski SK, Winterkorn EB, Paul E, Steele DJ, Halpern EF, Thiele EA: Renal manifestations of tuberous sclerosis complex: incidence, prognosis, and predictive factors. Kidney Int 2006, 70:1777-1782.

7. Nelson CP, Sanda MG: Contemporary diagnosis and management of renal angiomyolipoma. J Urol 2002, 168:1315-1325.

8. Bissler JJ, McCormack FX, Young LR, Elwing JM, Chuck G, Leonard JM, Schmithorst VJ, Laor T, Brody AS, Bean J, Salisbury S, Franz DN: Sirolimus for angiomyolipoma in tuberous sclerosis complex or lymphangioleiomyomatosis. N Engl J Med 2008, 358:140-151.

9. Davies DM, de Vries PJ, Johnson SR, McCartney DL, Cox JA, Serra AL, Watson PC, Howe CJ, Doyle T, Cross JJ, Tattersfield AE, Kingswood JC, Sampson JR: Sirolimus therapy for angiomyolipoma in tuberous sclerosis and sporadic lymphangioleiomyomatosis: a phase 2 trial. Clin Cancer Res 2011, 17:4071-4081.

10. Bissler JJ, Kingswood JC, Radzikowska E, Zonnenberg BA, Frost M, Belousova E, Sauter M, Nonomura N, Brakemeier S, de Vries PJ, Whittemore VH, Chen D, Sahmoud T, Shah G, Lincy J, Lebwohl D, Budde K: Everolimus for angiomyolipoma associated with tuberous sclerosis complex or sporadic lymphangioleiomyomatosis (EXIST-2): a multicentre, randomised, double-blind, placebo-controlled trial. Lancet 2013, 381:817-824.

11. Krueger DA, Northrup $H$ : Tuberous sclerosis complex surveillance and management: recommendations of the 2012 international tuberous sclerosis complex consensus conference. Pediatr Neurol 2013, 49:255-265.

12. Oesterling J, Fishman E, Goldman S, Marshall F: The management of renal angiomyolipoma. J Urol 1986, 135:1121-1124.

13. Steiner MS, Goldman SM, Fishman EK, Marshall FF: The natural history of renal angiomyolipoma. J Urol 1993, 150:1782-1786.

14. Johnson SR, Cordier JF, Lazor R, Cottin V, Costabel U, Harari S, Reynaud-Gaubert M, Boehler A, Brauner M, Popper H, Bonetti F, Kingswood C, Review Panel of the ERS LAM Task Force: European respiratory society guidelines for the diagnosis and management of lymphangioleiomyomatosis. Eur Respir J 2010, 35:14-26.

15. Northrup H, Krueger DA: Tuberous sclerosis complex diagnostic criteria update: recommendations of the 2012 international tuberous sclerosis complex consensus conference. Pediatr Neurol 2013, 49:243-254.

16. Bush A, Cramer D: Guidelines for the measurement of respiratory function. Respir Med 1994, 88:798.

17. Ryu JH, Hartman TE, Torres VE, Decker PA: Frequency of undiagnosed cystic lung disease in patients with sporadic renal angiomyolipomas. Chest 2012, 141:163-168.

18. Yamakado K, Tanaka N, Nakagawa T, Kobayashi S, Yanagawa M, Takeda K: Renal angiomyolipoma: relationships between tumor size, aneurysm formation, and rupture. Radiology 2002, 225:78-82.

19. van Baal JG, Smits NJ, Keeman JN, Lindhout D, Verhoef S: The evolution of renal angiomyolipomas in patients with tuberous sclerosis. J Urol 1994, 152:35-38.

doi:10.1186/s13023-014-0151-3

Cite this article as: Yeoh et al: Natural history of angiomyolipoma in lymphangioleiomyomatosis: implications for screening and surveillance. Orphanet Journal of Rare Diseases 2014 9:151. 